Results and Conclusion We found immigrants from Southern Asia to Western Asia had higher and immigrants from Eastern and Southerneastern Asia had lower risk of first MI than people born in Sweden. Within Europe, there seemed to be a slightly increasing risk trend from Southern and Western Europe to Eastern and Northern Europe. Low socioeconomic position was independent risk factor of first MI with education as the strongest predictor. However, we found occupation-based index might be more appropriate in immigrants study. Low education was also an independent risk factor for first MI survival. Both first MI risk and mortality decreased over the study period. Another novel finding was the surprisingly protective effect of with history of hyperlipidaemia or hypertension in the prognosis of first MI patients compared to those without.

\section{P1-378 WITHDRAWN}

\section{P1-379 PROJECTING PREVALENCE BY STAGE OF CARE FOR COLON CANCER AND ESTIMATING FUTURE HEALTH SERVICE NEEDS IN NEW SOUTH WALES AUSTRALIA}

doi:10.1136/jech.2011.142976f.70

${ }^{1,2} \mathrm{X} O \mathrm{Yu},{ }^{*}{ }^{3,4} \mathrm{M}$ Clements, ${ }^{1} \mathrm{D}$ O'Connell. ${ }^{1}$ Cancer Council, New South Wales, Sydney, Australia; ${ }^{2}$ The University of Sydney, Sydney, Australia; ${ }^{3}$ Australian National University, Canberra, Australia; ${ }^{4}$ Karolinska Institutet, Stockholm, Sweden

Aim To estimate the number of colon cancer patients in New South Wales (NSW) Australia requiring cancer care in the future.

Methods Prevalence of first primary colon cancer was estimated and projected forward, by stage of clinical care (initial care, long-term survivors, monitoring, and last year of life), for 2007-2016. Then using data from a patterns of care study for colorectal cancer, we estimated the type and amount of treatments that will be needed by colon cancer patients in each stage of care. Finally, data on metachronous tumours or second primary colon cancers were used to estimate the additional number of patients who will require care.

Results In NSW in 2006, there were 17687 patients aged 18-84 years alive with a past diagnosis of colon cancer. By 2016, this number will have increased to 22262 . The expected numbers of patients vary by stage of care with the majority in the monitoring stage $(41.4 \%)$ or being long-term survivors $(40.7 \%)$. There will be at least 1389 men and 1135 women requiring initial surgery for colon cancer in 2016. Of these 431 men and 352 women will also require adjuvant chemotherapy. In addition, there will be 741 patients with metachronous tumours or second primary colon cancers who will also need care in 2016 with $56 \%$ of them in the monitoring stage.

Conclusions These estimates reveal that in NSW the numbers of colon cancer patients requiring care for colon cancer are projected to rise by over $25 \%$ from 2006 to 2016.

\section{P1-380 SOCIAL NETWORK AND BINGE DRINKING IN SCHOOLS ADOLESCENTS}

doi:10.1136/jech.2011.142976f.71

\author{
${ }^{1,2} \mathrm{P}$ M Zarzar, ${ }^{*} \mathrm{~K} O \mathrm{~J}$ Jorge, ${ }^{1} \mathrm{E}$ Ferreira, ${ }^{1} \mathrm{M} \mathrm{P}$ Vale, ${ }^{2} \mathrm{~T}$ Oksanen, ${ }^{2} \mathrm{~K}$ Kawachi. \\ ${ }^{1}$ Universidade Federal de Minas Gerais, Belo Horizonte, Minas Gerais, Brazil; ${ }^{2}$ Harvard \\ School of Public Health, Boston, Massachusetts, USA
}

Introduction Hazardous alcohol drinking among the adolescent is of major concern. However, it is not known whether the type of social network is associated with binge drinking.

Methods We carried out a cross-sectional study among a random sample of 891 adolescents (40\% male, aged 15-19 years) from public to private schools in 2009-2010 in Belo Horizonte, Minas Gerais,
Brazil. Information on friendship network and binge drinking were collected using two validated self-administered questionnaires: the Integrated Questionnaire for the Measurement of Social Capital (CS-IO) and the Alcohol Use Disorders Identification Test (AUDIT). The Social Vulnerability Index (SVI) was used to assess area-level socioeconomic status. Multinomial regression analysis was used to estimate the associations between the type of most important friends and the frequency of binge drinking.

Results A total of 321 (36\%) adolescents reported binge drinking. We found no differences in binge drinking by age or gender whereas adolescents in private schools, in areas of less vulnerability and higher education of mother reported more binge drinking. Adjusted for age, sex and SVI, reporting friends from school rather than from church as the most important group of friends was associated with a higher likelihood of binge drinking at least monthly (OR 3.1 95\% CI 1.8 to 5.3) and at least weekly (OR $3.295 \%$ CI 1.4 to 7.2 ) when compared to never binge drinking, Lower socioeconomic status in the area was associated with lower likelihood of binge drinking. Conclusions Social network of adolescents is related to binge drinking.

\section{P1-381 THE IMPORTANCE OF SCHOOL-BASED PREVENTION STRATEGIES ON CARDIOVASCULAR HEALTH EDUCATION OF FAMILIES}

doi:10.1136/jech.2011.142976f.72

E Zera, ${ }^{*}$ K Sotirofski, K Leka. Regional hospital, Durres, Albania

Objective The goal of prevention programs is to reduce population exposure to environmental risk factors. Many people do not have sufficient knowledge about cardiovascular risk factors in Durres district.We used a school-based educational intervention program to promote the health knowledge in all participating families. The aim of study is to evaluate the school-based education intervention in improving cardiovascular risk factor knowledge of families.

Methods A total of 400 fifth-grade school children and their parents were invited in the study; 300 families were included in the study. The children attended a single session and were supplied with family information packages. Pre-and post-test questionnaires were completed by these families before and after the session. The questionaire aims to evaluate the knowledge collective not individual. There are questions to risk factor of $\mathrm{CHD}$, to nutrition and their contribute in CHD

Results There was a significant increase in the overall cardiovascular risk factor knowledge of families ( $p$ value $<0.001$ ). The parents of boys achieved a greater level of health knowledge in comparison to the parents of girls ( $p$ value $<0.005$ )

Conclusions This school-based educational intervention was effective in improving cardiovascular risk factor knowledge of families. Similar programs with more comprehensive methodology could be more effective in promoting healthy behaviours.

\section{P1-382 PREVALENCE OF ANAEMIA IN PATIENTS ADMITTED TO HOSPITAL WITH A PRIMARY DIAGNOSIS OF CONGESTIVE HEART FAILURE}

doi:10.1136/jech.2011.142976f.73

E Zera, ${ }^{*}$ A Hatellari. Regional hospital, Durres, Albania

Objectives To find the prevalence of anaemia in patients hospitalised with diagnosis of congestive heart failure (CHF) in our department of cardiology. 
Background There is growing evidence that anaemia is common in CHF and may contribute to the high morbidity and mortality of this condition. There is considerable disagreement about the prevalence of anaemia in this condition.

Methods In 219 consecutive patients were admitted to our hospital (January-December 2010) with diagnosis of CHF we extracted from the charts the haemoglobin $(\mathrm{Hb})$, serum creatinine, age, sex, NYHA functional class, smoking, diabetes, hypertension, hyperlipidaemia and cardiac aetiology of the CHF.Anaemia was considered when the $\mathrm{Hb}$ on admission was $<12 \mathrm{~g} / \mathrm{dl}$.

Results The patients were NYHA functional class III-IV.118 (53.8\%) of the 219 patients had a $\mathrm{Hb}$ on admission that was $<12 \mathrm{~g} / \mathrm{dl}$. The mean $\mathrm{Hb}$ for the entire group was $12.0+/-1.8 \mathrm{~g} / \mathrm{dl} .54(51.9 \%)$ of the 104 women were anaemic compared to 64 (55.6\%) of the 115 men. The mean serum creatinine was $1.8+/-1.2 \mathrm{mg} / \mathrm{dl}$. The prevalence of renal insufficiency (serum creatinine $>1.5 \mathrm{mg} \%$ ) was $48.5 \%$. There was a negative correlation between the level of serum creatinine and $\mathrm{Hb}(\mathrm{p}<0.00001$.) Of the 118 patients who were anaemic, most of 76 $(64.4 \%)$ had a serum creatinine $>1.6 \mathrm{mg} / \mathrm{dl}$.

Conclusions Anaemia is a common finding in patients hospitalised with CHF .In view of the negative effect of anaemia on cardiac function, it may be important contributor to the mortality and morbidity of CHF in these patients.

\section{OTHER THEMES}

\section{P1-383 SEXUAL BEHAVIOUR AND KNOWLEDGE OF PREVENTION OF SEXUALLY TRANSMITTED INFECTIONS AMONG STUDENTS IN CO-EDUCATIONAL AND NON CO-EDUCATIONAL SCHOOLS IN IBADAN, NIGERIA}

doi:10.1136/jech.2011.142976f.74

\begin{abstract}
A Adejimi, ${ }^{*}$ F Omokhodion, F Olaolorun. University College Hospital, Ibadan, Oyo State,
\end{abstract} Nigeria

Sexual interaction between students may be different in coeducational (CE) and non co-educational (NCE) schools. The objective was to compare sexual behaviour and knowledge of prevention of Sexually Transmitted Infections (STIs) among senior secondary school students in CE and NCE institutions in Ibadan. A comparative cross sectional study was carried out using a multistage sampling technique. There were 510 respondents; 250 from CE schools and 260 from NCE. We used semi-structured self administered questionnaires which included a 30-point STI knowledge scale with scores classified as good and poor. $\chi^{2}$ statistics was significant at $p<0.05$. The mean age of respondents was $15.9 \pm 1.5$ years, $47.5 \%$ were girls. The main sources of sex education were parents (44\%) and teacher (30.8\%). There were no significant differences between the two types of schools. Significantly higher proportion of girls in CE schools have had one sexual intercourse with the opposite sex (25.6\%-CE, 12.4\%-NCE) and had multiple sexual partners $(29.0 \%-\mathrm{CE}, 0 \%-\mathrm{NCE})$ compared with girls in NCE schools. Girls in NCE schools had significantly better knowledge of causes and prevention of STIs than those in CE schools $(28.8 \%-\mathrm{CE}$, $45.5 \%-\mathrm{NCE})$. There were no significant differences in the sexual behaviour and knowledge of causes and prevention of STIs among boys in the two types of schools. More girls in CE schools have had sexual intercourse compared to NCE schools whereas girls in NCE schools had better knowledge on sexually transmitted infections than those in CE schools. There is a need for strategies to increase reproductive health education in schools, particularly in CE schools.

\section{P1-384 INVERSE SAMPLING TO ESTIMATE DISEASE BURDEN OF LEPROSY IN INDIA: A PILOT STUDY}

doi:10.1136/jech.2011.142976f.75

A Aggarwal, * A Pandey. National Institute of Medical Statistics, New Delhi, India

Background and Objective Leprosy continues to be an important public health problem. The magnitude of the disease is often expressed by the number of cases registered by the system. Estimation of leprosy by conventional sampling is difficult due to large sample size requirement. A pilot study was conducted using inverse sampling in Bareilly district, Uttar Pradesh, India with an aim to evolve inverse sampling procedure vis-à-vis conventional sampling.

Method Two community development blocks one with low endemic area namely Fatehganj and other high endemic namely Ramnagar in Bareilly district of Uttar Pradesh, India were selected. Inverse sampling was adopted in Fatehganj while conventional cluster sampling was used in Ramnagar. Under inverse sampling, 25 new cases of leprosy were predetermined while a sample of 44000 subjects (population) was targeted for conventional sampling. Under inverse sampling the total population to be covered was not known in advance.

Findings A total of 63 new cases of leprosy were found in Ramnagar after covering a sample of 44686 population while a sample of 14734 population was covered in Fatehganj to detect 25 new cases of leprosy. Both the techniques provide similar estimates. The precision obtained under inverse sampling was though less than that under conventional sampling but found to be more feasible and suitable for estimation of leprosy due to less population covered, time and cost.

Conclusion Study reveals that inverse sampling was found to be advantageous over conventional sampling and could be adopted for the large scale survey at National level.

\section{P1-385 RISK FACTORS ASSOCIATED WITH LAST-MONTH ILLEGAL DRUG POLYCONSUMPTION IN A POPULATION OF DRUG USERS}

doi:10.1136/jech.2011.142976f.76

${ }^{1} \mathrm{~F}$ Alonso, ${ }^{1} \mathrm{M}$ L Garmendia, ${ }^{* 2} \mathrm{M}$ E Alvarado, ${ }^{3} \mathrm{G}$ Acuña. ${ }^{1}$ Universidad de Chile, Faculty of Medicina, School of Public Health, Santiago, Chile; ${ }^{2}$ Goverment of Chile, Conace, Santiago, Chile; ${ }^{3}$ Centro Nevería, Santiago, Chile

Introduction Drug polyconsumption is a negative predictor for treatment success and is related to increased risk-behaviour. However, little is known about risk factors associated to polyconsumption.

Objective To identify risk factors associated to last month illegal drugs polyconsumption (two or more drugs) in a Chilean population of adult drug users.

Methods In 2008 we administered a previously validated 6th version of the Addiction Severity Index to 436 adult drug users, recently admitted to rehabilitation programs, without serious psychiatric disorders and who signed an informed consent (age 32.5+9.3 years. $71.8 \%$ male). This semi-structured questionnaire includes information about: medical history, social support, employment, legal problems, drug use and psychiatry disorders. Last 30 days consumption was considered to calculate the number of drugs consumed by individuals entering a rehabilitation program. Multivariate logistic regression was used to identify risk factors.

Results Mean age at drug use initiation was $16+5.4$ y. Total number of drugs consumed ranged from 0 to 4 (theorical maximum 9). 30 days prevalence of polyconsumption was $30.5 \%$. Cocaine, marihuana and sedatives were the most common illegal drugs used 